\title{
Airway dysbiosis: Haemophilus influenzae and Tropheryma in poorly controlled asthma
}

Jodie L. Simpson ${ }^{1}$, Joshua Daly ${ }^{2}$, Katherine J. Baines ${ }^{1}$, Ian A. Yang ${ }^{3,4}$, John W. Upham ${ }^{3,5}$, Paul N. Reynolds ${ }^{6,7}$, Sandra Hodge ${ }^{6,7}$, Alan L. James ${ }^{8,9}$, Philip Hugenholtz ${ }^{2}$, Dana Willner ${ }^{2}$ and Peter G. Gibson ${ }^{1,10}$

Affiliations: ${ }^{1}$ Respiratory and Sleep Medicine, School of Medicine and Public Health, Centre for Asthma and Respiratory Disease, The University of Newcastle, Callaghan, Australia. ${ }^{2}$ Australian Centre for Ecogenomics, School of Chemistry and Molecular Biosciences and Institute for Molecular Bioscience, The University of Queensland, St Lucia, Australia. ${ }^{3}$ School of Medicine, The University of Queensland, St Lucia, Australia. ${ }^{4} \mathrm{Dept}$ of Thoracic Medicine, The Prince Charles Hospital, Chermside, Australia. ${ }^{5}$ Dept of Respiratory Medicine, Princess Alexandra Hospital, Woolloongabba, Australia. ${ }^{6}$ Dept of Thoracic Medicine, Royal Adelaide Hospital and Lung Research Laboratory, Hanson Institute, Adelaide, Australia. ${ }^{7}$ School of Medicine, The University of Adelaide, Adelaide, Australia. ${ }^{8}$ Dept of Pulmonary Physiology and Sleep Medicine, Sir Charles Gairdner Hospital, Nedlands, Australia. ${ }^{9}$ School of Medicine and Pharmacology, University of Western Australia, Crawley, Australia. ${ }^{10}$ Woolcock Institute of Medical Research, Glebe, Australia.

Correspondence: Jodie L. Simpson, Level 2, West Wing, Hunter Medical Research Institute, Lot 1 Kookaburra Circuit, New Lambton Heights, NSW 2305, Australia. E-mail: jodie.simpsonanewcastle.edu.au

ABSTRACT Asthma is a chronic inflammatory disorder of the airways where bacteria may act as protagonists of chronic inflammation. Little is known about the relation of airway inflammation to the presence of specific bacterial taxa. We sought to describe the sputum microbiome in adults with poorly controlled asthma.

DNA was extracted from induced sputum and microbial communities were profiled using 16S rRNA pyrosequencing. Bacterial species were characterised, and the relationship between microbial populations, asthma inflammatory subtypes and other covariates was explored. Real-time PCR was used to identify Tropheryma whipplei and Haemophilus influenzae in sputum.

Adults with neutrophilic asthma had reduced bacterial diversity and species richness. Tropheryma was identified and confirmed with real-time PCR in 12 (40\%) participants. Haemophilus occurred most often in a group of younger atopic males with an increased proportion of neutrophils. PCR confirmed the presence of $H$. influenzae in 35 (76\%) participants with poorly controlled asthma.

There are phenotype-specific alterations to the airway microbiome in asthma. Reduced bacterial diversity combined with a high prevalence of $H$. influenzae was observed in neutrophilic asthma, whereas eosinophilic asthma had abundant T. whipplei.

@ERSpublications

There are phenotype-specific alterations to the airway microbiome in asthma which may modulate local inflammation http://ow.ly/UbB9k

This article has supplementary material available from erj.ersjournals.com

Received: March 122015 | Accepted after revision: Oct 272015 | First published online: Dec 032015

Support statement: This research was supported by a project grant (569246) from the NHMRC Australia. Funding information for this article has been deposited with FundRef.

Conflict of interest: Disclosures can be found alongside the online version of this article at erj.ersjournals.com

Copyright OERS 2016 


\section{Introduction}

Asthma is a chronic heterogeneous inflammatory disorder of the airways involving eosinophilic and noneosinophilic inflammatory phenotypes. The mechanisms driving noneosinophilic airway inflammation and, in particular, neutrophilic inflammation are poorly understood.

A growing body of evidence supports a role for colonising bacteria in perpetuating inflammatory conditions in the lower airways $[1,2]$. The airway inflammatory response in neutrophilic asthma suggests bacterial activation with significantly upregulated Toll-like receptor 2 expression and increased interleukin (IL)- 8 and $-1 \beta$ levels compared with other inflammatory subtypes of asthma [3]. Patients with neutrophilic bronchitis are commonly culture-positive for Haemophilus influenzae $[1,2]$. These data suggest a potential role for the presence of lower airway bacteria, particularly $H$. influenzae, in the persistence of airway inflammation observed in neutrophilic asthma.

Technological advances such as culture-independent microbial community profiling make a detailed assessment of airway bacterial colonisation possible [4]. There may be an altered microbiome in the airways of patients with asthma, where bacteria of the phylum Proteobacteria (which include Haemophilus species) are more prevalent in asthma compared with healthy controls [4,5], particularly those with corticosteroid-resistant asthma [6]. The functional significance of these changes is unclear; however, associating bacterial colonisation with a typical host inflammatory response would provide stronger evidence for the role of airway dysbiosis in asthma. Little is known about the relationship between the airway inflammatory pattern that is specifically associated with the presence of specific bacterial taxa. In the present study, we used culture-independent microbial community profiling to characterise the lower airway microbiome in patients with and without asthma. Using these profiles, we explored the relationship between resident microbial communities and airway inflammatory phenotype. We hypothesised that patients with neutrophilic asthma would be characterised by a distinctive microbiome dominated by Haemophilus species compared with eosinophilic and paucigranulocytic asthma.

\section{Methods}

Participants

30 study participants (the initial cohort) were assessed with a full microbiome analysis (16S pyrosequencing) from four centres across Australia (Newcastle, New South Wales; Perth, Western Australia; and two centres in Brisbane, Queensland). An additional 16 adults with poorly controlled neutrophilic asthma were subsequently included in order to confirm the presence of $H$. influenzae using real-time PCR.

This study was conducted in accordance with the amended Declaration of Helsinki. Local institutional review boards approved the protocol (details in the online supplementary material) and written informed consent was obtained from all patients.

Asthma diagnosis was established using American Thoracic Society guidelines based on current episodic respiratory symptoms, doctor's diagnosis and evidence of variable airflow obstruction [7]. Participants with asthma were stable but symptomatic, despite being prescribed maintenance inhaled corticosteroid (ICS) treatment with an Asthma Control Questionnaire 6 (ACQ6) score $>0.7$ [8]. Participants had no reported exacerbations, infections or alterations in respiratory medications in the previous 4 weeks. Exclusion criteria and patient assessment details can be found in the online supplementary material. Nonsmoking healthy controls had normal lung function and no diagnosis of airway disease.

Design

All participants attended a single visit with pre- and post-bronchodilator spirometry and documentation of asthma symptoms, asthma-specific quality of life [9], medication use and smoking status assessed, and sputum induction performed.

\section{Sample collection}

Sputum induction with hypertonic saline (4.5\%) was performed as previously described [10]. Selected sputum was stored for DNA extraction or dispersed using dithiothreitol for sputum cell count assessment and inflammatory subtype determination [3].

DNA preparation, 16 S rRNA PCR, amplicon pyrosequencing and real-time PCR

Details of bacterial DNA preparation from samples, sequencing and sequencing data analysis along with the confirmatory real-time PCR methods are presented in the supplementary material.

\section{Data analysis}

Clinical data were analysed using Stata 11 (Stata, College Station, TX, USA) using standard methods as described in the online supplementary material. 


\section{Results}

\section{Clinical, inflammatory and typical culture profile}

Participants with neutrophilic asthma were slightly younger and commonly male, with similar lung function, asthma control score and asthma symptoms compared with those with non-neutrophilic asthma (table 1). Sputum inflammatory cell counts revealed significantly more total cells with an increased proportion of neutrophils and a reduced proportion of eosinophils, macrophages and columnar epithelial cells compared with the non-neutrophilic asthma group (table 1).

\section{Sputum microbiome}

Sputum samples from participants with neutrophilic asthma were less diverse and dominated by a small number of species (low richness and evenness), whereas the non-neutrophilic asthma participants had a diverse array of species and they were more evenly distributed in the community (high richness and evenness). This was shown by a significantly lower Shannon index, richness and Simpson index in adults with neutrophilic asthma (table 2). This difference was maintained after correction for age, gender, previous smoking and ICS dose (ANCOVA, $\mathrm{p}=0.035$ ).

Bacteria from the phyla Proteobacteria and Firmicutes (dominated by Streptococcus species) were most common, accounting for $>90 \%$ of the operational taxonomic units (OTUs) in the neutrophilic asthma group (table 2 and figure 1). There was a significantly lower proportion of OTUs for Actinobacteria and Firmicutes, and significantly more Proteobacteria in those with neutrophilic compared with non-neutrophilic asthma (table 2). The reduced richness and diversity in the neutrophilic asthma group appeared to be driven by a group of four participants with a high number of sequences for Haemophilus with very few other taxa, as shown in figure 1. There was no difference in the Shannon or Simpson index according to the study site

TABLE 1 Clinical and inflammatory cell parameters of participants with neutrophilic and non-neutrophilic asthma

\begin{tabular}{|c|c|c|c|}
\hline & Neutrophilic & Non-neutrophilic & p-value \\
\hline Subjects & 7 & 20 & \\
\hline Age years & $50.7 \pm 9.7$ & $60.3 \pm 10.8$ & 0.049 \\
\hline Male & $15(71)$ & $6(30)$ & 0.071 \\
\hline Atopic & $6(86)$ & $16(80)$ & 0.713 \\
\hline Ex-smoker & $2(29)$ & $11(55)$ & 0.224 \\
\hline Smoking pack-years & 1 and 24 & $16.8(2.2,56.5)$ & 0.621 \\
\hline FEV $1 \%$ predicted & $84.2(17.0)$ & $74.3(17.8)$ & 0.213 \\
\hline FEV $1 /$ FVC $\%$ & $74.8 \pm 7.5$ & $66.8 \pm 10.0$ & 0.064 \\
\hline ACQ6 score & $1.0 \pm 0.7$ & $1.4 \pm 1.0$ & 0.342 \\
\hline GINA treatment step & & & 0.562 \\
\hline 1 & $0(0)$ & $1(5)$ & \\
\hline 2 & $0(0)$ & $0(0)$ & \\
\hline 3 & $1(17)$ & $1(5)$ & \\
\hline 4 & $5(83)$ & $18(90)$ & \\
\hline Taking ICS & $6(86)$ & $18(90)$ & 0.610 \\
\hline ICS dose $\mu \mathrm{g}$ & $2000(800,2000)$ & $2000(1000,2000)$ & 0.933 \\
\hline \multicolumn{4}{|l|}{ VAS symptom scores } \\
\hline Breathlessness & $4.1(1.1,23)$ & $7.2(4.8,10.0)(n=19)$ & 0.341 \\
\hline Wheeze & $1.1(0.1,7.0)$ & $3.5(1.0,6.4)(n=19)$ & 0.469 \\
\hline Cough & $4.5(2.2,51)$ & $7.5(2.8,10.0)(n=19)$ & 0.665 \\
\hline Sputum production & $3.0(0.7,14.0)$ & $5.9(0.7,10.0)(n=19)$ & 0.729 \\
\hline \multicolumn{4}{|l|}{ Inflammatory cell counts } \\
\hline Total cell count $\times 10^{6} \mathrm{~mL}^{-1}$ & $7.2(6.7,9.1)$ & $3.2(2.1,5.0)$ & 0.015 \\
\hline Viability \% & $84(81,94)$ & $78(73,83)$ & 0.064 \\
\hline Neutrophils \% & $74.4 \pm 12.2$ & $30.0 \pm 16.7$ & $<0.001$ \\
\hline Eosinophils \% & $0.3(0.0,1.0)$ & $2.1(0.9,4.4)$ & 0.016 \\
\hline Macrophages \% & $21.5(14.8,34.8)$ & $52.7(41.6,68.9)$ & $<0.001$ \\
\hline Lymphocytes \% & $0.5(0.0,1.75)$ & $1.0(0.3,1.1)$ & 0.538 \\
\hline Columnar epithelial cells \% & $0.3(0.0,1.0)$ & $1.9(0.9,4.9)$ & $<0.001$ \\
\hline Squamous cells $\%$ & $1.2(0.0,11.3)$ & $4.7(1.4,8.6)$ & \\
\hline
\end{tabular}

Data are presented as $\mathrm{n}$, mean $\pm \mathrm{SD}, \mathrm{n}(\%)$ or median (quartile 1, quartile 3), unless otherwise stated. FEV1: forced expiratory volume in 1s; FVC: forced vital capacity; ACQ6: Asthma Control Questionnaire 6; GINA: Global Initiative for Asthma; ICS: inhaled corticosteroid; VAS: visual analogue scale. 


\begin{tabular}{|c|c|c|c|}
\hline & Neutrophilic & Non-neutrophilic & p-value \\
\hline Subjects & 7 & 20 & \\
\hline Shannon index & $1.1(0.9,4.2)$ & $3.8(3.4,4.2)$ & 0.010 \\
\hline Simpson index & $0.3(0.3,0.9)$ & $0.9(0.8,0.9)$ & 0.035 \\
\hline \multicolumn{4}{|l|}{ Bacteria phyla } \\
\hline Actinobacteria & $3.8(0.2,3.1)$ & $4.0(2.1,6.7)$ & 0.019 \\
\hline Bacteroidetes & $2.3(1.1,26.7)$ & $14.9(8.4,22.8)$ & 0.092 \\
\hline Firmicutes & $7.8(3.1,25.7)$ & $35.8(27.5,41.8)$ & 0.009 \\
\hline Fusobacteria & $0.5(0.3,3.8)$ & $3.7(2.3,7.8)$ & 0.103 \\
\hline Proteobacteria & $88.0(25.1,92.0)$ & $38.0(21.1,43.1)$ & 0.046 \\
\hline
\end{tabular}

Data are presented as $\mathrm{n}$ or median (quartile 1, quartile 3), unless otherwise stated.

where the sample was collected (online supplementary table S1). Dominant members of the microbial community included Prevotella, Streptococcus, Neisseriaceae and Haemophilus (figure 1).

\section{Smoking}

16 (53\%) participants had smoked in the past; this group tended to have a higher prevalence of sequences for bacteria from the phyla Actinobacteria and Bacteroidetes, and a lower prevalence of bacteria from phylum Proteobacteria compared with never-smokers. Bacteria from the phylum Fusobacteria were significantly more prevalent in ex-smokers compared with never-smokers. There was also a significant elevation in both the Shannon and Simpson indices in ex-smokers compared with never-smokers, indicating increased diversity in this group (table 3 ).

Smoking pack-years were significantly associated with the prevalence of Actinobacteria, $(\mathrm{r}=0.420 \mathrm{p}=0.021)$, but not with any of the other bacterial phyla. There was no association between smoking pack-years with either the Shannon or Simpson indices.

\section{Tropheryma whipplei}

Seven (23\%) participants had sequences corresponding to the genus Tropheryma (phylum Actinobacteria) in their induced sputum. Participants with Tropheryma sequences had similar age, lung function, ACQ6 score, visual analogue scale symptom score and sputum cell differential as those without OTUs for Tropheryma (table 4). In the seven participants where Tropheryma was identified, there was a significant negative association between Tropheryma OTU and wheeze scores (Spearman $\mathrm{r}=-0.821, \mathrm{p}=0.023$ ) (data not shown). Of note, five of the seven participants with Tropheryma also had eosinophilic airway inflammation.

Using PCR to screen for Tropheryma whipplei, 12 (40\%) participants were found to be positive: six participants identified from the original pyrosequencing analysis and an additional six newly identified samples. These data confirmed that the Tropheryma OTUs were in fact T. whipplei.

\section{Haemophilus influenzae}

Figure 2 shows the principal component analysis (PCA) and identified Haemophilus2 (OTU4) as a dominant microbe in a group of asthma participants. The clinical and inflammatory features of those with a high frequency of Haemophilus sequences (Haemophilus high) were then examined more closely. Haemophilus high participants were typically younger atopic males, who had never smoked, commonly of the neutrophilic asthma inflammatory subtype (table 5). Using univariate linear regression to examine other possible determinants of neutrophil proportion, there was no significant association between age, smoking pack-years, gender or dose of ICS. Participants in the Haemophilus high group had a 20\% higher sputum neutrophil proportion than other participants $(\mathrm{p}=0.074)$.

Using logistic regression, we asked which clinical and inflammatory outcomes were associated with a participant being in the Haemophilus high group. Age, sex and sputum neutrophil proportion were each independent predictors of participants being in the Haemophilus high group using univariate analysis. Smoking pack-years and dose of ICS were not associated with the Haemophilus high group.

Multivariate logistic regression revealed that a model including age, sex and sputum neutrophil proportion together predicted inclusion in the Haemophilus high group $\left(\mathrm{p}=0.0004 ; \mathrm{R}^{2}=0.599\right)$. None of the three 


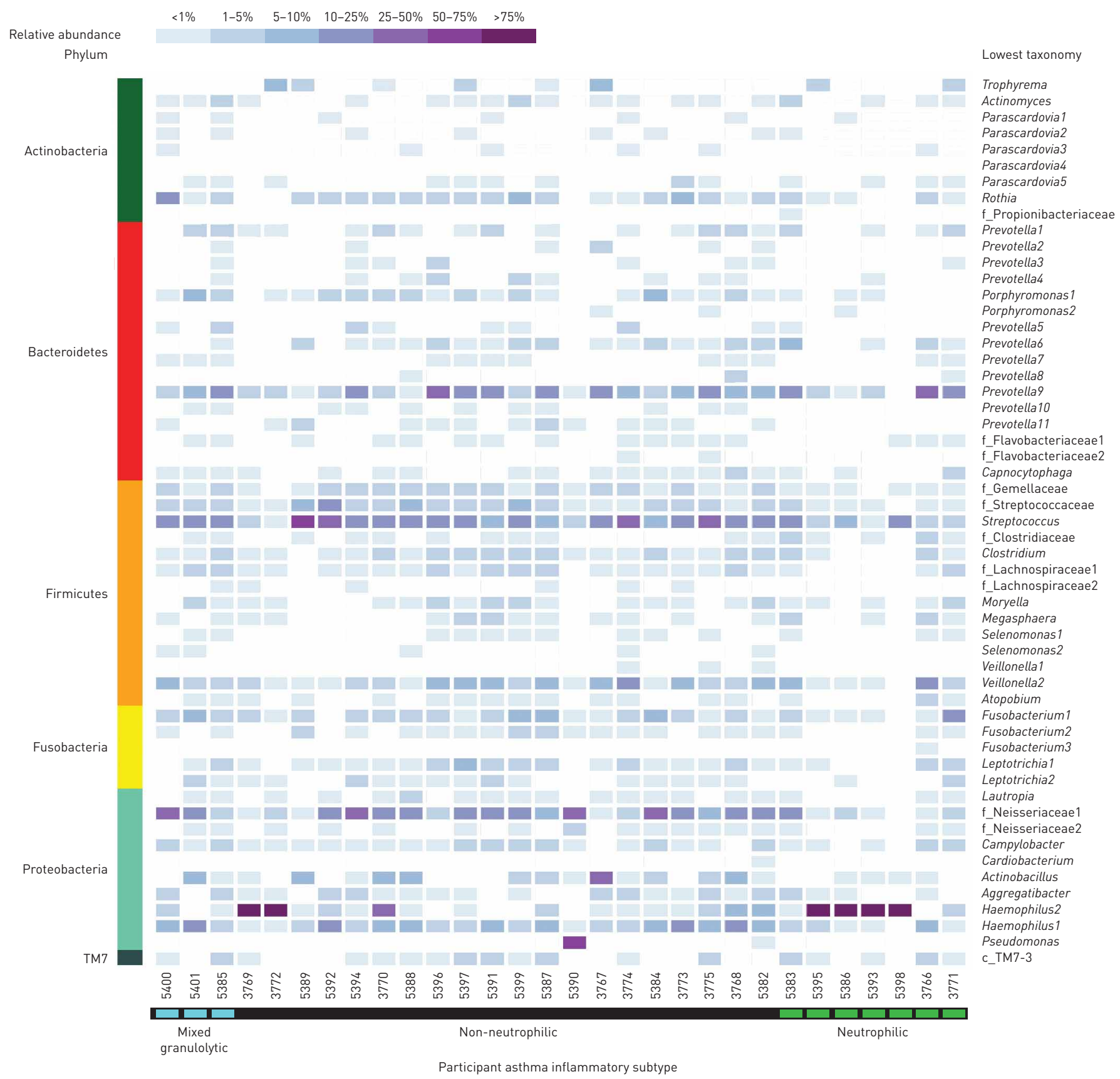

FIGURE 1 Heatmap showing the relative abundances of the dominant bacterial operational taxonomical units. f_: family; c_: class.

variables on their own predicted inclusion in the Haemophilus high group; however, this may be a power issue.

Given four of the six samples with a high abundance of Haemophilus OTU4 in the PCA were from a neutrophilic inflammatory subtype, we sought to enrich the number of neutrophilic asthma participants and determine if the species of Haemophilus was indeed $H$. influenzae. We identified a further 16 participants with poorly controlled neutrophilic asthma. Real-time PCR analysis was undertaken on all 46 participants (the initial cohort of 30 and the additional 16 with neutrophilic asthma) to identify $H$. influenzae. The clinical and inflammatory profiles of the further 16 participants are shown in online supplementary table S2.

24 of the 30 initial participants and 11 of the additional 16 neutrophilic asthma participants were PCR-positive for $H$. influenzae. The detection rate of $H$. influenzae was not different in those with neutrophilic and non-neutrophilic asthma ( $81 \%$ compared with $75 \%, \mathrm{p}=0.453)$. There were no significant 
TABLE 3 Sputum sample bacterial diversity and phyla for ex-smokers and never-smokers

\begin{tabular}{lccc} 
& Ex-smoker & Never-smoker & p-value \\
\hline Subjects & 16 & 14 & \\
Shannon index & $3.8(3.5,4.4)$ & $2.8(1.1,4.1)$ & 0.010 \\
Simpson index & $0.9(0.8,0.9)$ & $0.7(0.3,0.9)$ & 0.040 \\
Bacteria phyla & & & \\
$\quad$ Actinobacteria & $4.0(2.0,7.5)$ & $2.6(0.3,4.5)$ & 0.077 \\
Bacteroidetes & $14.5(8.4,23.9)$ & $9.1(1.1,22.9)$ & 0.299 \\
Firmicutes & $32.4(26.3,37.2)$ & $21.2(5.4,40.7)$ & 0.271 \\
Fusobacteria & $4.0(3.4,9.3)$ & $2.1(0.4,5.0)$ & 0.028 \\
Proteobacteria & $41.5(22.6,43.1)$ & $60.3(21.3,91.4)$ & 0.360 \\
\hline Data are presented as n or median (quartile 1, quartile 3), unless otherwise stated. & \\
\hline
\end{tabular}

differences in age, gender, atopy, lung function, ICS dose or smoking history between those who were PCR-positive or -negative for $H$. influenzae (data not shown). The ACQ6 score was significantly higher in those who were PCR-positive compared with those who were PCR-negative, mean \pm SD ACQ6 1.9 \pm 1.1 compared with $1.1 \pm 0.9, \mathrm{p}=0.037$.

\section{Discussion}

In the present study, adults with asthma had reduced bacterial diversity with the lowest diversity demonstrated in those with neutrophilic asthma. Dominant taxa were identified within specific inflammatory phenotypes. We identified a group of adult males with high abundance of Haemophilus sequences with predominantly neutrophilic airway inflammation. We also identified a group of participants with asthma who had Tropheryma sequences which we confirmed as T. whipplei. These participants most commonly exhibited an eosinophilic asthma subtype.

\begin{tabular}{|c|c|c|c|}
\hline & Tropheryma present & Tropheryma absent & p-value \\
\hline Subjects & 7 & 23 & \\
\hline Age years & $53 \pm 14$ & $60 \pm 9$ & 0.175 \\
\hline Male & 2 (29) & $10(43)$ & 0.403 \\
\hline Atopic & $5(71)$ & 19 (87) & 0.347 \\
\hline Ex-smoker & $4(57)$ & $12(52)$ & 0.581 \\
\hline Smoking pack-years & $16.7(7.7,47.8)$ & $14.4(1.3,40.3)$ & 0.505 \\
\hline FEV1 \% predicted & $81 \pm 24$ & $76 \pm 15$ & 0.577 \\
\hline FEV1/FVC \% & $71 \pm 10$ & $68 \pm 10$ & 0.517 \\
\hline ICS dose $\mu \mathrm{g}$ & $2000(2000,4000)$ & $2000(1000,2000)$ & 0.150 \\
\hline ACQ6 score & $1.0 \pm 0.7$ & $1.5 \pm 1.0$ & 0.250 \\
\hline \multicolumn{4}{|l|}{ VAS symptom scores } \\
\hline Breathlessness & $6.5(2.1,23.0)$ & $7.0(4.1,10.0)$ & 1.000 \\
\hline Wheeze & $3.5(1.0,4.6)$ & $3.2(0.8,6.7)$ & 0.683 \\
\hline Cough & $6.0(3.7,7.2)$ & $7.4(2.2,10.0)$ & 0.386 \\
\hline Sputum production & $3.8(0.0,5.9)$ & $4.7(1.8,10.0)$ & 0.740 \\
\hline \multicolumn{4}{|l|}{ Inflammatory cell counts } \\
\hline Total cell count $\times 10^{6} \mathrm{~mL}^{-1}$ & $5.2(3.3,7.2)$ & $4.6(2.2,8.9)$ & 0.713 \\
\hline Viability $\%$ & $85(74,94)$ & $81(75,84)$ & 0.328 \\
\hline Neutrophils \% & $40.9 \pm 25.5$ & $41.8 \pm 25.7$ & 0.942 \\
\hline Eosinophils \% & $1.8(1.0,7.0)$ & $1.5(0.3,4.0)$ & 0.769 \\
\hline Macrophages \% & $45(35,77)$ & $41(21,61)$ & 0.419 \\
\hline Lymphocytes \% & $1.0(0.3,2.5)$ & $0.5(0.3,1.0)$ & 0.457 \\
\hline Columnar epithelial cells \% & $0.5(0.3,2.5)$ & $1.3(0.3,3.0)$ & 0.475 \\
\hline Squamous cells $\%$ & $6.8(1.5,9.5)$ & $3.6(0.5,6.3)$ & 0.154 \\
\hline
\end{tabular}

Data are presented as $\mathrm{n}$, mean $\pm \mathrm{SD}, \mathrm{n}(\%)$ or median (quartile 1, quartile 3), unless otherwise stated. FEV 1 : forced expiratory volume in $1 \mathrm{~s}$; FVC: forced vital capacity; ACQ6: Asthma Control Questionnaire 6; ICS: inhaled corticosteroid; VAS: visual analogue scale. 


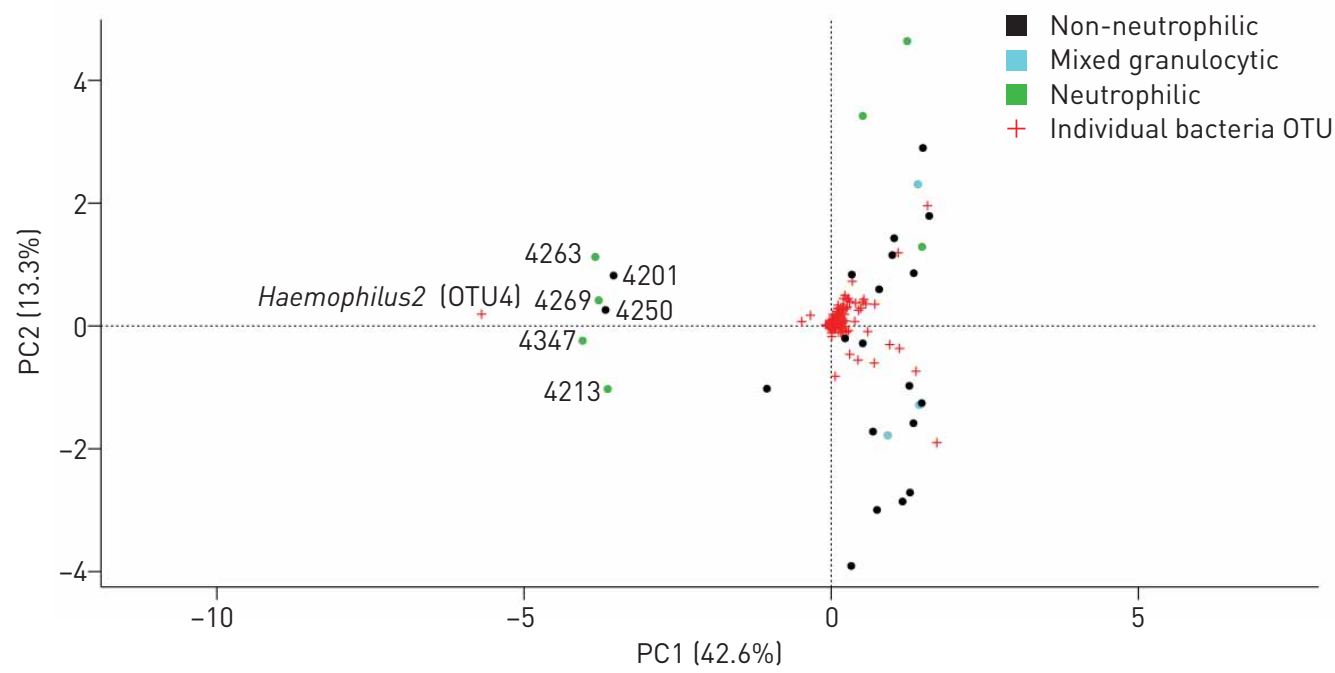

FIGURE 2 Principal component analysis plot. The axes represent each of the principal components (PC1 and PC2) and the proportion of the variation explained is shown in brackets. OTU: operational taxonomic unit.

Our data show reduced bacterial diversity in neutrophilic asthma. This is the first examination of bacterial diversity within asthma inflammatory subtypes. Diversity has been reported to be increased [5, 11] or similar [6] between patients with asthma and healthy controls. Similar variation in diversity has been reported in patients with chronic obstructive pulmonary disease (COPD) where bacterial diversity has been reported as increased [12] or reduced [13] compared with controls. As the majority of participants

TABLE 5 Clinical and inflammatory characteristics of participants with and without a high abundance of Haemophilus sequences (Haemophilus high and Haemophilus low groups)

\begin{tabular}{lccc} 
& Haemophilus high & Haemophilus low & p-value \\
\hline Subjects & 6 & 24 & \\
Age years & $45 \pm 14$ & $62 \pm 7$ & $<.001$ \\
Male & $5(83)$ & $7(29)$ & 0.026 \\
Atopic & $6(100)$ & $18(78)$ & 0.283 \\
Ex-smoker & $0(0)$ & $16(67)$ & 0.005 \\
Smoking pack-years & & $14.4(1.9,40.3)$ & \\
FEV1\% predicted & $82.2 \pm 14.2$ & $76.2 \pm 17.9$ & 0.452 \\
FEV1/FVC \% & $73.2 \pm 8.8)$ & $67.6 \pm 10.5$ & 0.241 \\
ICS dose $\mu$ g & $2000(2000,2000)$ & $2000(900,2000)$ & 0.393 \\
ACQ6 score & $1.19 \pm 0.68$ & $1.44 \pm 1.00$ & 0.571 \\
VAS symptom scores & & & \\
Breathlessness & $2.8(1.1,8.0)$ & $7.0(4.8,18.0)$ & 0.077 \\
Wheeze & $1.1(0.8,2.0)$ & $4.0(1.1,7.0)$ & 0.063 \\
Cough & $5.0(3.0,6.0)$ & $7.2(1.8,10.0)$ & 0.706 \\
Sputum production & $2.7(0.7,3.3)$ & $5.9(1.8,10.0)$ & 0.215 \\
Inflammatory cell counts & & & \\
Neutrophilic asthma & $4(67)$ & $3(15)$ & 0.024 \\
Total cell count $\times 10^{6}$ mL $^{-1}$ & $7.0(3.5,8.4)$ & $4.4(2.5,8.6)$ & 0.568 \\
Viability \% & $82(75,84)$ & $82(76,90)$ & 0.484 \\
Neutrophils \% & $61.2 \pm 33.6$ & $35.9 \pm 19.8$ & 0.027 \\
Eosinophils \% & $1.3(0.3,4.3)$ & $1.6(0.5,3.6)$ & 0.917 \\
Macrophages \% & $23.8(14.8,42.3)$ & $0.5(0.3,1.0)$ & 0.087 \\
Lymphocytes \% & $1.5(0.5,2.5)$ & $1.8(0.4,3.3)$ & 0.104 \\
Columnar epithelial cells \% & $0.4(0.3,1.0)$ & $3.7(1.1,7.0)$ & 0.152 \\
Squamous cells \% & $5.6(1.0,9.9)$ & & 0.622 \\
\hline Da & & & \\
\hline
\end{tabular}

Data are presented as $n$, mean $\pm S D, n(\%)$ or median (quartile 1 , quartile 3 ), unless otherwise stated. $F E V_{1}$ : forced expiratory volume in 1 s; FVC: forced vital capacity; ACQ6: Asthma Control Questionnaire 6; ICS: inhaled corticosteroid; VAS: visual analogue scale. 
were taking ICS, we are unable to determine if the use of ICS influences bacterial diversity. We corrected the diversity analysis for the dose of ICS, and found the difference in both Simpson and Shannon index was maintained after correction for ICS dose.

Studies of asthma in both adults and children report an increased frequency of sequences from the phylum Proteobacteria [4-6, 14]. HuANG et al. [14], in their study of 30 adults with severe asthma, observed that a worsening ACQ score was associated with an abundance of Proteobacteria and an association between T-helper 17-related gene expression (including CXCL8 and its receptors) with Proteobacteria taxa. We extend this to show this increase in Proteobacteria taxa is most prominent in those with neutrophilic asthma. We also observed significantly fewer OTUs from bacteria in the phyla Firmicutes and Actinobacteria in neutrophilic asthma. The anaerobic bacterial phylum Fusobacteria was also less frequent in neutrophilic asthma, although not statistically different. This phylum has been associated with corticosteroid-resistant asthma [15] which is known to be noneosinophilic in nature and suggests the absence of common oral anaerobes may be an important difference between asthma subtypes.

Currently, the impact of long-term ICS use on bacterial diversity is unknown. Early studies suggest that ICS use may influence the airway microbiome, resulting in a unique cluster of COPD patients taking ICS compared with those not taking ICS [12]. Similarly there was a unique cluster of taxa among those with corticosteroid-resistant asthma, including the genera Haemophilus and Tropheryma [15]. In this study, where the majority of participants were taking high doses of ICS, we also show a high prevalence of T. whipplei and $H$. influenzae in poorly controlled asthma. Little is understood about the impact of inhaled medications on oral flora. ICS may promote adverse oral effects; the most commonly reported of these is a higher rate of oral Candida species detection [16] and there is some evidence of mucosal damage in children with asthma [17].

This study is limited in its cross-sectional nature as it is not possible to distinguish between cause and effect. The strengths of this study include the well-characterised study population combining detailed clinical assessment with assessment of both airway inflammation and the airway microbiome. Induced sputum has been used frequently to study the airway microbiome [18-20]. We have attempted to reduce oral contamination by selecting the lower airway portions of the sample from the salivary material; however, oral contamination cannot be excluded. Other research suggests that while lower airway samples do contain an abundance of oral microbiota, there is a lung-specific signal where some OTUs are disproportionately found in bronchoalveolar lavage compared with oral wash, particularly Haemophilus species [21].

We identified seven patients with asthma who had sequences belonging to the genus Tropheryma (phylum Actinobacteria). The only named species of this genus, T. whipplei, causes Whipple's disease, a gastrointestinal disorder characterised by arthritis, diarrhoea and weight loss [22]. Increasing evidence supports a role for T. whipplei in airway disease with identification in interstitial lung disease [23], pneumonia [24, 25] and recently in corticosteroid-resistant asthma [6]. Pulmonary symptoms may precede gastrointestinal symptoms, including intermittent fever, dry cough and shortness of breath, which suggests that airway colonisation or infection may contribute to the symptoms and exacerbations observed in asthma. Our PCR confirmation resulted in a further five participants having evidence of T. whipplei, with a PCR detection rate of $40 \%$. Tropheryma has also been reported in asymptomatic HIV infection (13\%), specifically in the lung rather than the mouth [26], and this is further evidence that our identification of Tropheryma in samples of induced sputum may originate in the lower airway. The participants with Tropheryma tended to be taking a higher dose of ICS; however, this was not statistically different. While further research with longitudinal follow-up is needed to determine if this group of patients will develop Whipple's disease, identification of Tropheryma suggests previously unidentified microorganisms may be present in the airways, possibly contributing to asthma symptoms and airway inflammation.

$H$. influenzae is the most common species from the phylum Proteobacteria resulting in airway infection, and has been isolated from the airways of both adults and children with asthma $[1,27,28]$. In neutrophilic asthma, there is a high incidence of $H$. influenzae identified using traditional culture methods, compared with other inflammatory phenotypes [2]. In this study, we observed a group of adults with asthma with a predominance of Haemophilus OTUs who were younger, atopic, nonsmoking males who tended to have higher proportions of neutrophils. Since the sensitivity of the pyrosequencing method does not allow the exact species to be determined, we conducted real-time PCR using specific probes and found that $80 \%$ of participants with poorly controlled asthma were positive for H. influenzae. While PCR does not distinguish between the presence of dead and live organisms, it is important to recognise that even dead or degraded bacteria can elicit an immune response, e.g. bacteria endotoxin can induce significant inflammatory and immune signals. We have previously described high levels of sputum endotoxin in patients with neutrophilic asthma and, in the same group, a high incidence of positive culture for $H$. influenzae [2].

In conclusion, we have been able to link differences in the airway microbiome to altered host inflammatory response in asthma. There are phenotype-specific alterations to the airway microbiome in 
asthma. Reduced bacterial diversity combined with a high prevalence of $H$. influenzae was observed in neutrophilic asthma, whereas eosinophilic asthma had abundant T. whipplei. These changes may modulate local airway inflammation and immune responses. Understanding the relationships between these events will contribute to the development of more specific treatment strategies.

\section{Acknowledgements}

We wish to acknowledge the AMAZES research group who collected and processed the sputum samples, and Melinda Tooze and Bridgette Ridewood (The University of Newcastle, Callaghan, Australia) who undertook the confirmatory PCR.

\section{References}

1 Wood LG, Simpson JL, Hansbro P, et al. Potentially pathogenic bacteria cultured from the sputum of stable asthmatics are associated with increased 8-isoprostane and airway neutrophilia. Free Radic Res 2010; 44: 146-154.

2 Simpson JL, Grissell TV, Douwes J, et al. Innate immune activation in neutrophilic asthma and bronchiectasis. Thorax 2007; 62: 211-218.

3 Simpson JL, Phipps S, Baines KJ, et al. Elevated expression of the NLRP3 inflammasome in neutrophilic asthma. Eur Respir J 2014; 43: 1067-1076.

4 Hilty M, Burke C, Pedro H, et al. Disordered microbial communities in asthmatic airways. PLoS One 2010; 5 : e8578.

5 Marri PR, Stern DA, Wright AL, et al. Asthma-associated differences in microbial composition of induced sputum. J Allergy Clin Immunol 2013; 131: 346-52 e1-3.

6 Goleva E, Jackson LP, Harris JK, et al. The effects of airway microbiome on corticosteroid responsiveness in asthma. Am J Respir Crit Care Med 2013; 188: 1193-1201.

7 Standards for the diagnosis and care of patients with chronic obstructive pulmonary disease (COPD) and asthma. Am Rev Respir Dis 1987; 136: 225-244.

8 Juniper EF, O'Byrne PM, Guyatt GH, et al. Development and validation of a questionnaire to measure asthma control. Eur Resp J 1999; 14: 902-907.

9 Juniper EF, Guyatt GH, Ferrie PJ, et al. Measuring quality of life in asthma. Am Rev Respir Dis 1993; 147: 832-838.

10 Gibson PG, Wlodarczyk JW, Hensley MJ, et al. Epidemiological association of airway inflammation with asthma symptoms and airway hyperresponsiveness in childhood. Am J Resp Crit Care Med 1998; 158: 36-41.

11 Huang YJ, Nelson CE, Brodie EL, et al. Airway microbiota and bronchial hyperresponsiveness in patients with suboptimally controlled asthma. J Allergy Clin Immunol 2011; 127: 372-381.

12 Pragman AA, Kim HB, Reilly CS, et al. The lung microbiome in moderate and severe chronic obstructive pulmonary disease. PLoS One 2012; 7: e47305.

13 Erb-Downward JR, Thompson DL, Han MK, et al. Analysis of the lung microbiome in the "healthy" smoker and in COPD. PLoS One 2011; 6: e16384.

14 Huang YJ, Nariya S, Harris JM, et al. The airway microbiome in patients with severe asthma: associations with disease features and severity. J Allergy Clin Immunol 2015; 136: 874-884.

15 Goleva E, Hauk PJ, Hall CF, et al. Corticosteroid-resistant asthma is associated with classical antimicrobial activation of airway macrophages. J Allergy Clin Immunol 2008; 122: 550-559.

16 Fukushima C, Matsuse H, Tomari S, et al. Oral candidiasis associated with inhaled corticosteroid use: comparison of fluticasone and beclomethasone. Ann Allergy Asthma Immunol 2003; 90: 646-651.

17 del-Rio-Navarro BE, Corona-Hernandez L, Fragoso-Rios R, et al. Effect of salmeterol and salmeterol plus beclomethasone on saliva flow and IgA in patients with moderate-persistent chronic asthma. Ann Allergy Asthma Immunol 2001; 87: 420-423.

18 Huang YJ, Sethi S, Murphy T, et al. Airway microbiome dynamics in exacerbations of chronic obstructive pulmonary disease. J Clin Microbiol 2014; 52: 2813-2823.

19 Rogers GB, Zain NM, Bruce KD, et al. A novel microbiota stratification system predicts future exacerbations in bronchiectasis. Ann Am Thorac Soc 2014; 11: 496-503.

20 Lim YW, Evangelista JS III, Schmieder R, et al. Clinical insights from metagenomic analysis of sputum samples from patients with cystic fibrosis. J Clin Microbiol 2014; 52: 425-437.

21 Morris A, Beck JM, Schloss PD, et al. Comparison of the respiratory microbiome in healthy nonsmokers and smokers. Am J Respir Crit Care Med 2013; 187: 1067-1075.

22 Whipple G. A hitherto undescribed disease characterized anatomically by deposits of fat and fatty acids and mesenteric lymphatic tissues. Bulls Johns Hopkins Hospital 1907; 18: 383-393.

23 Harris JK, De Groote MA, Sagel SD, et al. Molecular identification of bacteria in bronchoalveolar lavage fluid from children with cystic fibrosis. Proc Natl Acad Sci U S A 2007; 104: 20529-20533.

24 Bousbia S, Papazian L, Auffray JP, et al. Tropheryma whipplei in patients with pneumonia. Emerg Infect Dis 2010; 16: 258-263.

25 Fenollar F, Ponge T, La Scola B, et al. First isolation of Tropheryma whipplei from bronchoalveolar fluid and clinical implications. J Infect 2012; 65: 275-278.

26 Lozupone C, Cota-Gomez A, Palmer BE, et al. Widespread colonization of the lung by Tropheryma whipplei in HIV infection. Am J Respir Crit Care Med 2013; 187: 1110-1117.

27 Kloepfer KM, Lee WM, Pappas TE, et al. Detection of pathogenic bacteria during rhinovirus infection is associated with increased respiratory symptoms and asthma exacerbations. J Allergy Clin Immunol 2014; 133: 1301-1307.

28 Zhang Q, Illing R, Hui CK, et al. Bacteria in sputum of stable severe asthma and increased airway wall thickness. Respir Res 2012; 13: 35. 